\title{
Spin as a determinable
}

\author{
J. Wolff \\ final draft \\ please cite the published version: TOPOI April 2015 \\ DOI: $10.1007 / \mathrm{s} 11245-015-9319-2$
}

\begin{abstract}
In this paper I aim to answer two questions: 1) Can spin be treated as a determinable? 2) Can a treatment of spin as a determinable be used to understand quantum indeterminacy? In response to the first question I show that the relations among spin number, spin components and spin values cannot be captured by a single determination relation; instead we need to look at spin number and spin value separately. In response to the second question I discuss three ways in which the determinables model might be modified to account for indeterminacy, and argue that none of them is fully successful in helping us to understand quantum indeterminacy.
\end{abstract}

\section{Introduction}

In this paper I explore the possibility of capturing quantum indeterminacy by treating quantum properties, like spin, as indeterminate determinables. While I will argue that this route of dealing with indeterminacy is not entirely successful, a careful investigation is nonetheless useful, both to stress the difficulties in accounting for quantum indeterminacy, and to raise some general concerns for the attempt to treat quantum properties on the determinable model. To assess the prospects for such approaches to quantum indeterminacy, two questions need to be answered. First, can observables, like spin, be characterized as determinables? Secondly, can the usual model of determinables and their determinates be modified in such a way as to accommodate indeterminacy arising from superposition states for quantum observables?

The motivation for investigating these two questions arises from two sources. On the one hand, properties in quantum mechanics are not very well understood. The determinable/determinate model is one of the most commonly used ways of understanding classical physical quantities like mass (e.g. Hawthorne [2008], Dasgupta [2013]), so a natural move would be to turn to this model for the case of quantum properties as well. On the other hand, recent work on metaphysical indeterminacy has turned to the relation between determinables and determinates as a way of characterizing metaphysical indeterminacy [Wilson, 
2013]. Applying this model to cases of quantum indeterminacy arising from the superposition of quantum properties, specifically in the case of spin-states, allows us to explore both the status of properties in quantum mechanics, and the nature of quantum indeterminacy.

I will begin by introducing some assumptions I will be making for the purposes of this paper. This is necessary since quantum indeterminacy is only understood as a metaphysical indeterminacy on some interpretations of quantum mechanics. I will also introduce certain assumptions about the distinction between determinates and determinables. On this basis I go on to characterize some elementary features of spin, and develop a way of applying the determinable/determinates model to a vectorial quantity like spin. I then explore and assess three possible ways of tweaking the determinate/determinable distinction to allow for quantum indeterminacy. Finally I briefly look at the case of entanglement.

\section{Background assumptions}

To set up the questions of how to treat spin and what to make of quantum indeterminacy, we need to make some assumptions about quantum mechanics. These assumptions are by no means uncontroversial, and I do not claim to have offered a defense for them here.

The first such assumption is that quantum mechanics suggests metaphysical indeterminacy, not (merely) epistemic indeterminacy. Metaphysical indeterminacy appears to be a consequence of 'orthodox' (Dirac - von Neumann) readings of quantum mechanics. At the heart of this orthodox reading is the idea that a quantum state is represented by a ray in a Hilbert space, and that this representation is complete. Orthodox quantum mechanics assumes that a system is in an eigenstate for some observable $O$ iff that observable $O$ takes on one of its eigenvalues. While some observables are compatible in the sense that a system can at the same time be in eigenstates with respect to these observables (e.g. the Cartesian components of the momentum operators), many observables are incompatible. This is true in particular position and momentum, and of the different components of spin. When a system is in a state of superposition with respect to some observable $O$, the system has no eigenvalues with respect to that observable. ${ }^{1}$

Not all interpretations of quantum mechanics accept the eigenstate-eigenvalue link, or the definition of a quantum state as a ray in a Hilbert space. Bohmians assign a definite position to each particle at all times, while recovering experimental results by introducing what is clearly intended as an epistemic indeterminacy [Dürr et al., 2013]. As a result, Bohmian accounts typically do not treat quantum states as involving metaphysical indeterminacy. For the purposes of this paper I shall set aside accounts, like the Bohmian, which avoid metaphysical

\footnotetext{
${ }^{1}$ For a more detailed technical discussion of quantum indeterminacy on orthodox interpretations, see Bub [2000].
} 
indeterminacy altogether. Instead I will focus on more orthodox interpretations, which at least suggest that the indeterminacy is not just epistemic.

Another aspect in which I will be addressing more orthodox approaches to quantum mechanics is the treatment of spin (and mass, and charge) as properties. Some contemporary approaches to quantum mechanics deny that spin, mass, and charge should be treated as intrinsic properties, ${ }^{2}$ or even go so far as to suggest that there are no properties in the fundamental ontology of quantum mechanics [Esfeld, 2014]. Without settling the matter between defenders of these positions and more orthodox accounts, I will assume here that spin, mass, and charge can at least prima facie be treated as properties, and that we can accordingly ask what constitutes a good metaphysical model for such properties.

Since metaphysical indeterminacy is often seen as an obvious downside for orthodox quantum mechanics, the question whether we can use metaphysical tools to make sense of it, seems quite pressing. If it turned out that metaphysical indeterminacy is well-understood in the case of orthodox quantum mechanics, the puzzles associated with orthodox quantum mechanics would be considerably reduced. Conversely, if philosophical accounts of metaphysical indeterminacy are unable to cope with indeterminacy arising in orthodox interpretations of quantum mechanics, non-orthodox accounts would seem more appealing.

Attempts to make precise the notion of ontic indeterminacy usually involve the idea that metaphysical indeterminacy is a matter of being 'undecided' or 'unsettled' between two or more definite conditions (see for example Williams and Barnes [2011]). What is indeterminate, according to these accounts, is which determinate state of affairs obtains. George Darby [2010] has argued that such accounts of metaphysical indeterminacy fail to capture quantum indeterminacy. Jessica Wilson [2013] has called such approaches 'meta-level' approaches, and contrasts them with 'object-level' approaches, which instead understand ontic indeterminacy as the determinate obtaining of an indeterminate state of affairs. An indeterminate state of affairs, according to her suggestion, is one in which an object instantiates a determinable property, but instantiates either none of its determinates, or multiple of its determinates. In this paper I will only be concerned with object-level attempts of understanding quantum indeterminacy.

In addition to these restrictions in the interpretations of quantum mechanics and metaphysical indeterminacy, I will make some assumptions about the nature of the determinable/determinate distinction. I will proceed on the assumption that determinables and determinate are properties (not just predicates), which stand in special relationships to one another. While some philosophers have suggested that at least fundamentally, only determinate properties exist [Gillet and Rives, 2005], I will assume for the purposes of this paper that both determinables and determinates exist. This assumption is required to articulate at least one of the responses to superposition states, namely the idea that a

\footnotetext{
${ }^{2}$ For arguments against intrinsic properties in quantum mechanics see Ladyman and Ross [2007], for a defense of intrinsic properties in fundamental physics see Ney [2010]. Since the question of whether quantum properties are determinables is independent of the question whether they are intrinsic, I won't be discussing these arguments in detail.
} 
determinable can be instantiated while none of its determinates is instantiated.

My starting point will be a fairly standard contemporary characterization of the relationship between determinables and determinates, as laid out by Funkhouser [2006, 548-9]:

1. properties are determinable or determinate only relative to other properties

2. determinate properties are specific ways of having determinable properties

3. no instantiation of a determinable without instantiation of one of its determinates

4. instantiation of a determinate necessitates instantiation of all relevant determinables

5. determination is transitive, asymmetric, irreflexive

6. determinates under the same determinable admit of a special kind of comparison

7. the chain of determinables-determinates is finite

This list of characteristic relations derives from the original home of the determinable/determinate distinction as a model of the relations among color predicates. The following may serve as a quick illustration of points 1-5: red is a determinable relative to scarlet, and a determinate relative to colored; scarlet is a specific way of being red, crimson another. Something cannot be red without being a determinate shade of red, like crimson or scarlet, and anything that is crimson is automatically also red and colored. If scarlet determines red, and red determines colored, then scarlet also determines colored.

The final two points are somewhat more controversial, but the idea is as follows: I can compare different shades of red with respect to how similar they are, and order them by similarity. Maroon is more similar to cardinal than either is to scarlet, and all three are more similar to each other than any of them are to navy. Moreover, we would not know how to compare colors and shapes in an analogous way, i.e., how to compare being red to being triangular. And there is an end to how finely I can differentiate shades of color: there are different shades of red, but no different shades of crimson. ${ }^{3}$

The determinables/determinate distinction can be applied not only to monadic properties, but to relations as well. For example, the relation ' $\mathrm{x}$ is $\mathrm{n}$ times larger than $y$ ' is a determinable with determinates ' $\mathrm{x}$ is three times larger than $\mathrm{y}$ ', ' $\mathrm{x}$ is five times larger than y', and so forth. So the mere fact that a property is

\footnotetext{
${ }^{3}$ This last point seems a lot more controversial when we think of determinates as properties than when we think of them as predicates. How fine-grained our color predicates are would seem to be a matter of how finely human perception can draw distinctions between colors. By contrast, whether there are further differentiations of crimson, beyond what humans can distinguish, seems to be a matter of what's true about color, not color terms. Since this won't matter for the case of spin, though, I will set this worry aside.
} 
non-monadic is no reason to think the determinable model cannot be applied. This will matter since spin, as well as other relevant properties, are sometimes thought to be in some sense relational.

To sum up, for the purposes of this paper I will be addressing orthodox interpretations of quantum mechanics, which suggest genuine metaphysical indeterminacy. I will discuss object-level attempts at understanding this indeterminacy, which apply a modified determinable/determinate structure to quantum observables like spin.

\section{Modeling spin as a determinable}

For the purposes of this paper I will primarily be concerned with electron spin. Spin is understood as something like the internal angular momentum of elementary particles, which endows these particles with a small magnetic dipole

moment. Electrons are spin $1 / 2$ particles, in contrast to bosons, which have integer spin numbers.

The most metaphysically neutral way of describing spin is to look at a SternGerlach experiment. In a Stern-Gerlach device (SGD) silver atoms are sent through an inhomogeneous magnetic field and afterwards detected on a screen. The pattern on the screen reveals that the atoms are deflected in the passage through the magnetic field: half of the atoms are sent 'up' and the other half 'down'.

This basic Stern-Gerlach experiment shows that spin is discrete, further experiments with iterated, but differently oriented Stern-Gerlach devices show that different components of spin (along different axes) are incompatible. In particular, a measurement of spin along one axis seems to 'erase' spin-values measured along a different axis. For example, suppose we measure the spin along the 'z-axis' and get the (usual) result of half of the atoms being deflected upwards, half downwards. The probability for spin-values 'up' and 'down' respectively along an axis perpendicular to $\mathrm{z}$, is $1 / 2$, that is, were we to measure the spin-values of the atoms along the 'y-axis' next, we would once again see half of them being deflected upwards, half downwards. But in carrying out that measurement, information about the previous values for the $\mathrm{z}$-direction would be lost, and we could once again only assign a probability of $1 / 2$ for each value in the z-direction. The particle is said to be in a superposition state with respect to spin along the 'z-axis'.

Mathematically these relations are expressed by the three operators

$$
\begin{aligned}
& \mathbf{S}_{\mathbf{x}}=1 / 2\left(\begin{array}{ll}
0 & 1 \\
1 & 0
\end{array}\right) \\
& \mathbf{S}_{\mathbf{y}}=1 / 2\left(\begin{array}{cc}
0 & -i \\
i & 0
\end{array}\right) \\
& \mathbf{S}_{\mathbf{z}}=1 / 2\left(\begin{array}{ll}
1 & 0 \\
0 & 1
\end{array}\right)
\end{aligned}
$$


Since the eigenvectors of each operator form a complete basis of a Hilbert space for spin- $1 / 2$, the respective eigenvectors of the other two operators can be expressed as linear combinations of the first. In our experiment, when we've measured spin in the z-direction, thereby determining the spin-eigenstates for $\mathbf{S}_{\mathbf{z}}$, the eigenvectors for $\mathbf{S}_{\mathbf{x}}$ and $\mathbf{S}_{\mathbf{y}}$ will be expressed as linear combination of the eigenvectors of $\mathbf{S}_{\mathbf{z}}$.

Accordingly the basic features of spin a metaphysical model should capture are:

- that spin numbers sort particles into kinds

- that the different components of spin are incompatible; measuring spinvalues for one component leaves the electrons in a state of superposition between the respective spin values of the orthogonal components

- that the possible spin values for each component are discrete

Since having a particular spin number sorts particles into kinds, it is tempting to provide an analysis of spin in analogy with common ways of understanding quantities like mass and charge. Electrons have a characteristic mass and charge, as well as a characteristic spin, which differentiates them from other particles. On standard accounts of mass in metaphysics [Hawthorne, 2008], mass is thought of as a determinable, and particular masses, like $1 \mathrm{~kg}, 10 \mathrm{~kg}, 100 \mathrm{~kg}$, as determinates of that determinable. It would be neat if spin turned out to be analyzable along similar lines.

Before we can ask what to do about superposition states, we need see how the determinables model can be applied to spin in the first place. While the application is straightforward in the case of scalar magnitudes like mass, it is less obvious how the model applies to vector quantities in general, and vector-like quantities such as spin in particular. Vectors are quantities with a magnitude and a direction. Typical vectors in classical physics include force, acceleration, and momentum. Spin, like these vector quantities, has a direction, but unlike them, the directedness of spin is not a spatial direction in ordinary space (hence the description of spin as an 'internal angular momentum'). Whether the direction is understood spatially or not, we can distinguish two elements of direction: orientation and sense [Massin, 2009]. ${ }^{4}$

A vector is fully determinate when its direction and its magnitude are completely specified. For example, a car speeding up at $3 \mathrm{~m} / \mathrm{s}^{2}$ along the direction from Edinburgh to Glasgow has a determinate acceleration. The magnitude is given by $3 \mathrm{~m} / \mathrm{s}^{2}$, the orientation by reference to the direction from Edinburgh to

\footnotetext{
${ }^{4}$ Two caveats. First, the mere fact that a quantity can be mathematically represented by a vector does not entail that it is directed-position can be represented through vectors, but it is not directed (for additional discussion see Beisbart [2009]). Second, the distinction between sense and orientation seems to pertain primarily to dynamical quantities, like force, and less obviously to purely kinematic ones, like velocity. I do not have space here to pursue this question in more detail, but I don't think it will make a difference for the discussion of spin.
} 
Glasgow, and the sense by noting that the speed increases rather than decreases. What are the appropriate determinables?

Since vectors have a magnitude and a direction, the determinable model has to be applied separately to each part. We should, on the one hand, treat the scalar part of a vector as one determinable, while keeping the direction fixed. The determinates for the scalar part of a vector are vectors of different magnitude, but the same direction. For example, the car might be speeding up at a rate of $4 \mathrm{~m} / \mathrm{s}^{2}$, or of $2 \mathrm{~m} / \mathrm{s}^{2}$. Provided it is traveling in the same direction as before, these are different determinates for the same determinable.

For the direction part of the vector we have to distinguish two aspects: sense and orientation. Each vector has some orientation (in virtue of being a vector), the determinables in question are different particular orientations. Since at least for classical vectors orientations are spatial, orientations require appropriate spaces to be represented; for the vectors of classical physics the spaces are typically real-valued. Moreover, to determine particular orientations, as opposed to merely stating that vectors are oriented in some way, we need a reference system with respect to which the vector can be said to be oriented. The need for reference frames introduces a concern about conventionality or observer dependence for classical vectors, since there is typically no reason to think that a particular reference frame is to be preferred over all others. As a result, different specifications of the same vector quantity, from the perspective of different reference frames, seem equally valid (see Beisbart [2009] for discussion). Even though the full specification of a classical vector quantity is dependent upon the introduction of reference frames, it is nonetheless the case that each such specification can be unproblematically understood as a complete specification of the state. This is not true in the same way for the case of spin.

Because vectors have directedness, and especially because they have sense, Massin [2009] and others have argued that they must be understood as relational, since reference to other entities is needed to attribute a directed property to an object (but see Busse [2009] for a defense of vectors as intrinsic). Without deciding the matter in the general case, it seems that in the case of spin, this worry does not arise. Since the directedness of spin is non-spatial, it is not directedness towards another object in the sense in which force is directed at another object. Accordingly, the mere fact that spin has directedness does not make it relational; instead spin seems like a good candidate for an intrinsic property. Reasons for regarding spin as extrinsic derive from spin entanglement states, not from its directedness.

Nonetheless, because spin has a vector like structure, the application of the determinable model is far from straightforward. We can distinguish spin number, spin component and spin value. Spin numbers behave somewhat like the magnitude component of classical vectors, and the determinables model applies to them fairly straightfowardly: spin number is a determinable, integer spin numbers and half-spin numbers are its determinates, their determinates in turn are spin 1 , spin 0 , and spin $1 / 2 .{ }^{5}$ To find the orientation of spin we need to

${ }^{5}$ Why not $+1,-1$, and $+1 / 2$ and $-1 / 2$ ? Because the sign in these cases represents the 
look at the different spin components, and it is here that matters become difficult. For spin, like for other vectors, these components behave in some respects like determinables, but they behave differently from components for spatially oriented vectors. The relevant components are the operators introduced above, and the relevant vector spaces are Hilbert spaces. The three operators do not commute, and each pair their of eigenvectors forms a complete basis for the Hilbert space. Finally we have spin value, which is a determinable with the two determinates 'up' and 'down'.

A maximally determinate spin for a given electron is, for example, $S_{z}=$ $+1 / 2$, or spin-up in the $\mathrm{z}$-direction for a spin- $1 / 2$ particle. Two features distinguish this maximally determinate spin-state from the state of maximally determinate acceleration. First, electrons are in such a fully determinate spin state only in relatively rare circumstances, e.g. after passing through an appropriately oriented Stern-Gerlach Device. Secondly, when a spin-state is maximally determinate for one of the operators, that is, when it is in an eigenstate with respect to the observable represented by that operator, it is maximally indeterminate between spin values for the remaining two observables. That means not only are particles rarely in maximally determinate spin states, they are at best in maximally determinate spin states with respect to one component of spin.

We are now in a position to formulate the problem of quantum indeterminacy for the case of spin in terms of determinables and determinates. The problem concerns the relationship between spin-components and their determinates, and spin-values and their determinates. A spin value depends, for its determination, on a choice of operator, since a determinate spin value can be ascribed to a particle only if it is in the eigenstate of one of the spin operators. Since the operators are mutually exclusive, this furthermore means that the spin values with respect to the remaining components will not be determinate. Spin, unlike classical vector quantities, is never fully determinate.

\section{How to deal with indeterminacy}

With an understanding of how the determinables/determinate model might be applied to spin, we can look at particular ways to modify the distinction in ways that account for indeterminacy. Three possible ways of incorporating the indeterminacy of spin-values into the model of spin as a determinable have been discussed in the literature, although none of these options has been fully explored.

The first strategy is to allow for the probabilistic having of determinates, and has been suggested, in passing, by Funkhouser [2006]. The other two strategies are discussed in Wilson [2013], in the context of using the determinable/determinate model to understand metaphysical indeterminacy in general. Wilson proposes, on the one hand, that determinables can be instantiated while several of their determinates are instantiated, and on the other hand that determinables might be instantiated without any of their determinates being

'sense' of the vector, which I discuss separately. 
instantiated. The latter, she suggests, might be applicable to quantum indeterminacy. I shall consider all three options here, and outline the problems I see with applying each of them to the case of spin. Of these three possibilities, I'll argue that treating superpositions as a case of a determinable without determinates is the most promising, but even so it remains less than fully satisfying.

The first strategy is prima facie a minimal departure from the traditional understanding of determination relations. Funkhouser suggests that "an amendment for the quantum level might be that every object instantiating a determinable also instantiates certain determinates to certain probabilities" [Funkhouser, 2006,566 , note]. One way to understand the idea that the determinates themselves might be probabilistic is to look at the 'spin vector' which can be expressed as a vector the components of which are the expectation values $\left\langle S_{x}\right\rangle$, $\left.<S_{y}\right\rangle,\left\langle S_{z}\right\rangle$. Since the expectation values are indeed linked to probabilities, one might try to understand them as determinates of a determinable which takes values between 0 and 1 . The trouble is that the probabilities themselves are not determinate spin values. The determinate spin values are still 'up' and 'down' (or '+' and '-'), not, for example, 0.4 and 0.6. What exactly the probabilities denote is of course controversial, but minimally they simply state the likelihood of finding a particle with spin value 'up' and down respectively in a given direction. By adding in the probabilities, we simply seem to acknowledge the indeterminacy of the spin state, we don't eliminate it. ${ }^{6}$ Indeed, Funkhouser's own framework for understanding determinables as property spaces makes it clear why quantum properties cannot be accounted for in that framework. Funkhouser suggest that a determinable forms a property space, where a property space is defined as "[t]he metaphysical space that covers some range of physically compossible determination dimension values (along with the corresponding non-determinable necessities) for a network or any participation of such a network" [Funkhouser, 2014, 26]. The components of spin do not form physically compossible determination dimensions; only one of their values can be determinate at any given moment. This is not by itself an objection to Funkhouser's framework, but it is clear that quantum properties cannot be understood as determinables in that framework.

Since the first strategy for understanding spin as a determinable while allowing for indeterminacy through spin-superposition does not work, we may have to look to a more radical revision of the determinables model to find a solution. Jessica Wilson proposes two different departures from the standard model, each of which allows for a certain amount of indeterminacy in the instantiation of determinables.

In the first case we might allow that a determinable can be multiply realized, where the multiple realizations of the determinable are relativized to different perspectives. Suppose the determinable in question is colored. Usually

\footnotetext{
${ }^{6}$ Assuming, as I have been throughout the paper, that the probabilities cannot simply be understood as measures of our ignorance about the spin state of the particle. And of course we do have good reason to believe that as long as we follow the orthodox interpretation of the quantum state as fully represented by a ray in a suitable Hilbert space, probabilities cannot be understood as a measure of our ignorance. See Bub [2000] for discussion.
} 
we assume that if the determinable colored is instantiated, exactly one of its determinates, say red, is instantiated. How could color be indeterminate in the relevant sense? Wilson suggests iridescent feathers, which are definitely colored, but which color they are depends on the angle from which they are viewed.

It is relatively easy to see how this proposal might be applied to the case of vector quantities in general. Different perspectives would correspond to different reference frames, and the particular determinations of direction in those different reference frames correspond to the particular determinate shades of green or blue we attribute to the feather from different angles. If your assessment and my assessment of the determinate color of the feather differs, because we are viewing it from different angles, neither of our perspectives is 'more correct' than the other. Similarly, for two observers with different states of motion, the specification of a vector quantity may differ, without it being the case that one of them is uniquely correct.

While Wilson does not apply this option to the quantum case, a natural way of extending this reading to the case of quantum properties would be to think of projections to the eigenstates of different components of quantum mechanical observables as different, complementary representations. For the case of electron spin this would mean that we treat the determinate outcomes of spin measurement in different directions as different perspectives. Depending on which measurement we carry out, i.e., how we orient our Stern-Gerlach device, we will get a determinate z-spin up, say, or a determinate y-spin down, but it would be misleading to suggest that the electron only has a determinate z-spin or only a determinate y-spin. It is just that from the perspective (read: measurement) we have chosen, this is the determinate which is realized in our perspective.

There are three concerns for this approach. First, the perspectival reading of complementarity suggests that the different eigenstates of an observable, understood as different determinates of the same determinable, could be instantiated at the same time, but would need to be 'looked at' from different perspectives. This looks too much like an epistemic reading of quantum mechanical uncertainty, not a reading that allows for metaphysical indeterminacy. The uncertainty would be due to our limitations: we are forced to carry out one measurement rather than a different one, but the spin-state is understood as determinate independent of which measurement we choose to carry out. Obviously such an epistemic reading may well be ultimately correct, but since we set out to find a metaphysical model for quantum indeterminacy, it seems the perspectival model falls short of this promise.

Secondly, suppose two electrons pass through a Stern-Gerlach device, oriented along the 'z-axis', and one of them goes up, the other down. It would seem that both have a determinate spin value along the z-axis, but they have different determinates for z-spin. Both are in superposition states with respect to spin along orthogonal axes. But the relationship between the two definite spin-values and the complementarity with respect to spin-values along other axis seems to be of a different sort. Spin values 'z-spin up' and 'z-spin down' would have to be understood as mutually exclusive determinates of $\mathrm{z}$-spin, in 
the same way in which crimson and scarlet are mutually exclusive determinates of red. That would just be the usual idea that at most one determinate of each determinable is instantiated. Spin values ' $\mathrm{x}$-spin up' and ' $\mathrm{x}$-spin down' are determinates of a different determinable, namely $\mathrm{x}$-spin. Switching perspective in this case would simply be looking at different determinables. The color case was supposed to be one of looking at the simultaneous instantiations of multiple determinates of the same determinable.

What seems surprising in this case is not that the different determinates exclude one another, but that there seems to be a relationship between the different determinables such that the determination of one determinable precludes determination of a different determinable. Again, the mathematical description of spin allows us to describe quite precisely what's going on here, but finding determinables that mutually exclude one another seems highly unusual. It is a bit like saying that something has a shape and a color, but if it has a determinate shape it does not also have a determinate color and vice versa.

A third, related difficulty is that in the case of iridescent feathers, we still think that if we switch perspectives, we can 'recreate' the same experience as before, that is, we think that repeated 'measurements' after switching perspectives are possible. Moving back and forth between different angles, the feather's color will vary systematically. By contrast, once the orientation of the SternGerlach Device has been switched, there is once again a fifty percent chance of obtaining a spin-value up or down with respect to the newly chosen axis. Previous spin-measurements along orthogonal axes cannot be recovered. Having multiple determinates realized does not help, then. What about the idea of determinables without determinates?

The third strategy, according to which a determinable may be instantiated without instantiation of any of its determinates, seems more promising for handling indeterminacy. Before the interaction with a SGD, electrons definitely have spin, but it would seem they do not have determinate spin values for the different spin components. They are in a superposition state. Moreover, it seems as though the setting of the SGD determines, for which of the spin-components spin-values will be determined. After the electrons have passed through the apparatus, they have determinate spin-values for the measured spin-direction. So we might say that here is a physical process, which determines the previously indeterminate determinable.

Assigning a determinate spin value to a particle in a particular direction necessarily leaves the spin values of that particle in other directions indeterminate. We are still forced to say that a particle with a determinate z-spin up has indeterminate spin values for $\mathrm{x}$-spin and $\mathrm{y}$-spin. Permitting the instantiation of determinables without determinates helps to describe this phenomenon, because we can say that $\mathrm{x}$-spin and $\mathrm{y}$-spin are determinables with two determinates each, and that neither of these determinates is instantiated even though the determinables 'spin- $x$ ' and 'spin-y' are.

But while this gives us an attractive model to work with, there are some residual worries. Spin, like other quantum properties, seems special in that it requires a particular physical intervention (a measurement/measurement-like 
interaction) to become determinate. Why are certain processes, but not others, suitable for bringing about the determination? That is to say, the quantum measurement problem remains unsolved (which is perhaps as it should be).

What is more puzzling, and not fully explained by the model at hand, is the fact that different determinables should be so related as to make joint determination impossible. Why is it that a full determination of spin value in a particular direction precludes simultaneous determination in a different direction? Once again, it's not the fault of the model that this is so, but it shows that even if the model successfully captures certain features of spin, it does not provide a much better understanding of them.

Of the three answers to the question of indeterminacy, then, the third seems to be the most promising. It is also the most radical revision of the determinables/determinate distinction, since it requires the instantiation of determinables without determinates. If that is to be possible, determinables have to be accepted into the ontology on equal footing with determinates. ${ }^{7}$ It is not obvious that this is a price worth paying, given how little the application of the determinables model seems to contribute to our understanding of quantum indeterminacy. Even if fundamental determinables are useful in other branches of science, as Wilson [2012] suggests, the peculiar way in which determinables mutually exclude determination in quantum mechanics still seems quite puzzling. Of course, if non-quantum properties could be shown to have a similar structure, we might well find the model to be illuminating. So whether we should revise the determinables/determinates model to apply to the case of spin and other quantum properties will depend upon whether the model proves useful elsewhere as well.

\section{Entanglement}

So far I have discussed problems for applying the determinate/determinable model to spin and to the indeterminacy of spin state superpositions. In doing so I have only talked about single particles. This was natural, since determinables and determinates are usually thought to have fine-grained instantiations. Notice for example, that the rule that only one determinate per determinable may be instantiated makes sense only if we assume that determinates are instantiated in (smallish) parts of objects. Footballs are black and white, just not black and white allover.

The smallest unit of instantiations has to be small enough to instantiate only a single determinate. In the case of continuous determinables, like colors, that means we have to assume point-like instantiations of determinates. For quantities like charge, the typical assumption is that the minimal instantiation is any carrier of unit charge. In the case of spin this would suggest that we

\footnotetext{
${ }^{7}$ Wilson [2012] suggests that we should indeed do so, because determinables (and not just determinates) seem to play important roles in science, for example in laws of nature and in the special sciences. She also argues that standard objections to determinables as fundamental entities can be resisted by a friend of fundamental determinables.
} 
should take individual particles to be the minimal instantiators of spin.

It is not clear, however, that this will work for all cases. Notoriously, a joint spin-state is ascribed to an entangled pair of particles, but no definite spin-state can be assigned to either particle in the entanglement. We've already seen that the determinables model struggles to incorporate the indeterminacy involved in such a case, and that our best bet is to go with the idea that no determinates are instantiated in cases of superpositions. Can we apply the solution to the case of entanglement as well?

For an entangled state the smallest unit of instantiation is a pair of electrons, not a single electron. This is not surprising, of course, but it creates additional problems for the determinate/determinable model. For throughout the standard discussion of that model, it is assumed that the same unit of instantiation can instantiate determinables as well as determinates. The case of entangled particles suggests that determinables of the form 'x-spin', 'y-spin', 'z-spin' are instantiated by individual particles as well as entangled pairs, and ensembles of suitably prepared particles. At first glance this would seem to be no more remarkable than the fact that color can be instantiated by a jar of jelly beans, as well as individual jelly beans. The remarkable fact about spin entanglement is that the instantiation of determinables and determinates in the different particles is not independent. Once the spin value for a particle is determinate along a particular axis, the spin value of the other particle along that axis has to be opposite.

To apply the determinables/determinate model, modified along the lines of strategy three above, to entangled particles, it seems we have to say that not only are determinables sometimes instantiated without determinates, but that they are sometimes instantiated as holistic properties, whereas determinates are instantiated locally, i.e. by a single particle. Moreover, unlike classical determinables, which only require the instantiation of exactly one determinate, the determinables of quantum mechanics require the instantiation of both determinates, provided the determinable had previously been instantiated by a pair of particles. As before, the model doesn't explain why this might be the case, but it offers a possible way framing the issue in terms of determinables.

\section{Conclusion}

I set out in this paper to answer two questions, first whether the determinate/determinable model can be applied to spin, and secondly whether quantum indeterminacy can be illuminated using this model. My answer to both questions has been skeptical. The determinable/determinate model can be applied to spin only step-wise, that is, separately to spin number and spin value. This is due simply to the fact that spin is a vector like quantity. Restricting the view just to the question of determinate spin values, I then discussed three ways of understanding the indeterminacy of spin values. I suggested that of the strategies discussed, the proposal that determinables can be instantiated without their determinates seems to be the most promising. Accepting this proposal 
requires not only a radical revision in our metaphysical understanding of the determinable/determinate distinction, it also leaves questions open about indeterminacy in quantum mechanics. How much this model ultimately contributes to our understanding of quantum indeterminacy will depend upon finding cases of determinable/determinate relations exhibiting a similar structure, but from the non-quantum realm.

\section{References}

Claus Beisbart. How to fix directions or: Are assignments of vector characteristics attributions of intrinsic properties? Dialectica, 63(4), 2009.

Jeffrey Bub. Indeterminacy and Entanglement: The Challenge of Quantum Mechanics. The British Journal for the Philosophy of Science, 51:597-615, 2000.

Ralf Busse. Humean Supervenience, Vectorial Fields, and the Spinning Sphere. Dialectica, 63(4):449-489, 2009.

George Darby. Quantum Mechanics and Metaphysical Indeterminacy. Australasian Journal of Philosophy, 88(2), 2010.

Shamik Dasgupta. Absolutism vs comparativism about quantity. Oxford Studies in Metaphysics, 8, 2013.

D. Dürr, S. Goldstein, and N. Zanghì. Quantum Physics without Quantum Philosophy. Springer, 2013.

Michael Esfeld. Quantum Humeanism, or: Physicalism without properties. The Philosophical Quarterly, 64(256), 2014.

Eric Funkhouser. The Determinable-Determinate Relation. Noûs, 40(3):548$569,2006$.

Eric Funkhouser. The logical structure of kinds. Oxford University Press, 2014.

Carl Gillet and Bradley Rives. The nonexistence of determinables: Or, a world of absolute determinates as default hypothesis. Noûs, 39(3):483-504, 2005.

John Hawthorne. Quantity in Lewisian Metaphysics. In Metaphysical Essays, pages 229-237. Oxford University Press, 2008.

James Ladyman and Don Ross. Every thing must go. Oxford University Press, 2007.

Olivier Massin. The Metaphysics of Forces. Dialectica, 63(4):555-589, 2009.

Alyssa Ney. Are there fundamental intrinsic properties? In Allan Hazlett, editor, New Waves in Metaphysics, chapter 11, pages 219-239. Palgrave Macmillan, 2010. 
J. Robert G. Williams and Elizabeth Barnes. A Theory of Metaphysical Indeterminacy. Oxford Studies in Metaphysics, VI, 2011.

Jessica M. Wilson. Fundamental determinables. Philosophers' Imprint, 12(4), 2012.

Jessica M. Wilson. A determinable-based account of metaphysical indeterminacy. Inquiry, 56(4):359-385, 2013. 